\title{
Impact of home spirometry on medication adherence among adolescents with cystic fibrosis
}

\author{
Aarti Shakkottai MD ${ }^{1}$ | Niko Kaciroti PhD ${ }^{1}$ | Lauren Kasmikha ${ }^{1}$ | \\ Samya Z. Nasr MD, CPI ${ }^{1}$
}

1 Pediatric Pulmonology, Michigan Medicine, Ann Arbor, Michigan

2 Department of Biostatistics, University of Michigan School of Public Health, Ann Arbor, Michigan

\section{Correspondence}

Samya Z. Nasr, MD, CPI, Department of Pediatrics and Communicable Diseases, Michigan Medicine, 1500 E. Medical Center Drive, SPC 5212, Ann Arbor 48109-5212, MI. Email: snasr@umich.edu

Funding information

University of Michigan Charles Woodson

Biostatistics Fund; Cystic Fibrosis

FoundationThird Year Fellowship Training,

Grant number: SHAKKO15AO

\begin{abstract}
Objective: Medication adherence among adolescents with cystic fibrosis (CF) is often suboptimal and this has significant impact on their health and quality of life. The purpose of the study was to evaluate the impact of frequent home pulmonary function (PFT) monitoring on medication adherence among adolescents with CF.

Hypothesis: We hypothesized that weekly home PFT monitoring will improve adherence while not significantly adding to the treatment burden.

Methods: Individuals aged 12-21 years with CF were provided a spirometer to measure PFTs weekly for 1 year. Results were reviewed weekly via telephone. PFT data were downloaded from the device during quarterly clinic visits. Adherence was calculated from prescription refill data and compared to the previous year. Perceptions of treatment burden were assessed using the CF questionnaire-revised (CFQ-R) quality of life measure. Health outcome measures including nutritional status and PFTs from clinic were collected for the study period and the year prior.

Results: Thirty-nine subjects participated in the study. Mean age was $15.89 \pm 2.18$ years and $54 \%$ were female. Mean adherence to weekly spirometry monitoring was $59.47 \pm 24.60 \%$. Values generated on the device showed good correlation with those obtained in clinic. Mean medication possession ratio (MPR) was $60 \%$ in the previous year and $65 \%$ during the study $(P=0.04)$. Mean treatment burden scaled score on the CFQ-R was 68 at enrollment and 66 at study completion $(P=0.14)$.

Conclusions: Frequent home PFT monitoring is feasible in CF adolescents and could successfully improve medication adherence without significantly impacting treatment burden.
\end{abstract}

\section{KEYWORDS}

adolescents, cystic fibrosis, home spirometry, medication adherence, medication possession ratios, prescription refill histories, pulmonary function tests

\section{1 | INTRODUCTION}

Adherence to medications is often suboptimal among patients with chronic diseases like cystic fibrosis (CF). Data from electronic medication monitoring devices and from analysis of prescription refill histories reveal adherence rates of $30-75 \%$ among individuals with CF. ${ }^{1-10}$ Adolescents have significantly lower rates of medication adherence compared to other age groups. ${ }^{2,3,5,8}$ Prescription refill data over a 5-year period revealed adherence rates of $46-65 \%$ to pancreatic enzymes, vitamins, inhaled hypertonic saline (IHS), and dornase alfa 
among patients with CF 13-21 years of age. The same study showed adherence rates of $73-83 \%$ among those $0-5$ years of age. ${ }^{2}$ A different study evaluated adherence using electronic pill caps and also found lower rates of adherence to vitamins among adolescents compared to those less than 12 years of age ( $57 \%$ vs $85 \%){ }^{8}$

Individuals with CF have a high clinical, psychosocial, and economic burden that is compounded in the setting of nonadherence. ${ }^{1-5}$ Adolescents are particularly vulnerable since there is a significant decline in pulmonary function (PFT) that occurs during this time. ${ }^{11,12}$ An important risk factor for this reduction in PFT is having frequent pulmonary exacerbations, ${ }^{13}$ a factor that has been linked to poor medication adherence. ${ }^{1,3,4}$ Poor nutritional status is another risk factor for worsening PFTs among children and adolescents with CF. ${ }^{13,14}$ Those who demonstrate good medication adherence have also been noted to have better weight gain and higher body mass indices (BMI) irrespective of their age. ${ }^{2,7}$

Barriers to medication adherence among adolescents with CF include forgetfulness, competing time pressures, and doubts about the necessity, or usefulness of the prescribed treatment regimen. ${ }^{11,15-25}$ There is a decline in parental supervision during adolescence, which has been shown to negatively impact treatment adherence in this group. ${ }^{22}$ Knowledge about the disease, which is often deficient among adolescents, has also been shown to influence adherence. ${ }^{23}$ With worsening disease severity, the complexity of the prescribed treatment regimen increases in patients with $\mathrm{CF}^{25-27}$ More treatments are added in an effort to slow down the progression of the disease. This escalation in treatment complexity has been shown to adversely affect perceptions of treatment burden, with the effects being more pronounced among adolescents. ${ }^{25-27}$ Having a high perception of treatment burden can negatively impact self-management skills and lower adherence. ${ }^{11,16,19,24-26}$

Many of the same studies that elicited the aforementioned barriers have also identified multiple adherence motivators such as recognition of the impact of non-adherence on health outcomes, a strong relationship with the CF care team and encouragement from friends and family. ${ }^{11,15-17,19-23,25,26}$ Interventions that promote selfmanagement skills, whether they be a knowledge-based intervention such as providing more education about the disease process and available treatments, or a behavioral intervention like motivational interviewing, appear to improve medication adherence in individuals with CF. ${ }^{11,16,19,25-26,28-35}$ The use of technology to promote selfmanagement skills and adherence is becoming increasingly popular. ${ }^{30-35}$ Medication reminders have been successful in enabling patients with CF to take ownership of their disease and have resulted in improved adherence. ${ }^{11,19,31-33}$ However, very little data, exists on the impact of these interventions on the already high perceptions of treatment burden.

Reviewing PFT results appear to be an adherence motivator for many adolescents and young adults. ${ }^{17,19,34,35}$ Home PFT monitoring has enabled clinicians to obtain more frequent data and allows patients the opportunity to readily review and keep track of the results. ${ }^{17,36,37}$ Lechtzin et $\mathrm{al}^{37}$ conducted a large, randomized controlled trial evaluating the benefits of early detection of pulmonary exacerbations on PFT in adults with CF (mean age of 27 years). Participants assigned to the intervention arm were asked to measure their PFTs and fill out an online respiratory symptom diary twice a week from home for a 1-year duration. Both of these were viewed in real time by an electronic monitoring system. Any new symptoms or significant declines in PFT were identified as potential pulmonary exacerbations and immediately reported to the care team so an acute/sick clinic visit could be scheduled. In contrast, participants in the usual care arm were asked to call the clinic with symptoms and schedule acute/sick visits as needed for potential exacerbations.

Despite having twice as many acute/sick visits for pulmonary exacerbations and earlier detection times, the intervention group did not experience a slower rate of a decline in PFT compared to the controls. ${ }^{37}$ Hospitalization rates were also similar between the groups. ${ }^{37}$ Not unexpectedly, perceptions of treatment burden were higher in the intervention arm. ${ }^{37}$ While several outcome measures were evaluated in the study, medication adherence was not one of them. To our knowledge, this is the first study to evaluate the role of frequent home spirometry in promoting medication adherence.

This was a prospective interventional study investigating the impact of frequent home PFT monitoring on medication adherence, perceptions of treatment burden, and health outcomes among adolescents with CF. We hypothesized that providing adolescents with CF the opportunity to measure their PFTs from home would result in improved medication adherence. We also hypothesized that once a week monitoring of PFTs would not significantly affect perceptions of treatment burden.

\section{2 | METHODS}

Patients with CF 12-21 years of age were invited to participate in the study. Participants had to have a confirmed diagnosis of CF with a sweat chloride measurement $\geq 60 \mathrm{mEq} / \mathrm{L}$ and/or two disease-causing mutations and they had to be clinically stable for at least 1 month prior to enrollment. Eligible participants were approached during routine clinic visits and provided with information regarding the study. Those who were interested arranged to meet with the study team after their clinic visit. Participants were recruited between July and December 2015 and everyone who was invited agreed to participate in the study. Written informed consent and/or assent was obtained from all participants and/or their parents/legal guardians. The study was approved by the University of Michigan Institutional Review Board (IRB).

All participants received a Spiro $\mathrm{PD} \circledast$ personal spirometer to monitor their PFTs once a week from home for 12-months. The Spiro $\mathrm{PD} \otimes(P M D$ Healthcare in Allentown, PA), is a FDA approved device. It generates flow-volume loops and volume-time curves and reports the forced vital capacity (FVC), forced expiratory volume in $1 \mathrm{~s}$ (FEV1) and forced expiratory flow between $25 \%$ and $75 \%$ (FEF25-75) as a percent predicted using NHANES III reference values. PFTs can be stored and tracked on the device and downloaded as a PDF. 
Participants were instructed on the correct use of their device by a respiratory therapist (RT) at enrollment. The RT also ensured that the demographic information for each participant was accurately entered into the device. Time was also allotted for participants to practice using their device while the RT was still present. Participants were contacted weekly by telephone to address any questions or concerns with the device and record PFT results. These telephone calls, which had a specified script, were made by research assistants and the research coordinator and were designed primarily to check in with the participants to ensure they were not having any difficulties with the device. The PFT data documented through the telephone calls were also meant to serve as a backup, in case there were any technical difficulties with retrieving the data from the device. The participants determined the date and time of the telephone calls. Some of them arranged to call the research coordinator at a predetermined time every week while others chose to communicate via text messages. All subjects met with the study team during their quarterly clinic visits to review technique, address potential questions and concerns, and download PFT data from the device.

Adherence was monitored using prescription refill data. A medication possession ratio (MPR) was calculated for IHS, dornase alfa, pancreatic enzyme replacement therapy (PERT), and CF vitamins for the 12-month study period and the 12-months prior to enrollment. A MPR is calculated by dividing the number of days a medication was dispensed by the total number of days the medication was prescribed, within a 365-day period. These four medications were chosen since they are all routinely prescribed to patients with CF and could provide valuable information about differences in adherence between inhaled and oral medications. ${ }^{38,39}$ Participants were notified at enrollment that their adherence would be monitored and were asked to provide a list of pharmacies from where they receive the aforementioned medications. Permission was obtained from the participants and/or their parents/ legal guardians to contact the pharmacies for prescription refill data. The pharmacy list was verified and updated as necessary at each quarterly visit and upon study completion. Of note, while the weekly telephone calls addressed barriers to use of the Spiro $\mathrm{PD} \circledast$, it did not enquire about medication adherence or potential barriers to adherence.

Perceptions of treatment burden were assessed based on responses to the CF questionnaire-revised (CFQ-R), a wellvalidated, health-related quality of life measure that is specific to individuals with CF. ${ }^{40}$ Participants filled out the CFQ-R at enrollment and end of study. Their responses were used to calculate a scaled treatment burden score between 0 and 100 for each time point, with higher scores signifying lower perceptions of treatment burden. Results of PFTs obtained in clinic, BMI percentiles, and frequency of pulmonary exacerbations requiring either oral, or intravenous antibiotics were obtained through review of the medical records. Values obtained during the study period were compared to those from the year prior.

Participants received monetary compensation and had the option of keeping their Spiro PD® at the end of the study.

\section{1 | Statistical methods}

The Poisson regression model was fitted using generalized estimating equations to account for within subject correlation to estimate the change in adherence from that in the 12-months prior to enrollment to that during the study across the four medications. A MPR was calculated for the study period and the year prior to enrollment using the ratio between total days' refilled, or the total number of days within a 365-day period when the subject was in possession of the medication, and total days' prescribed, for each of the four medications. Within the Poisson regression model, the total days' refilled was used as the outcome and the total days' prescribed was used as the offset variable.

The intra class correlation coefficient (ICC) was used to assess the reliability of the FEV1 percent values generated on the device with those obtained in clinic. A repeated measures analysis using mixed models was used to model the trajectory of changes in the BMI percentile and FEV1 percent predicted from the 12-months prior to enrollment to the 12-month study period. An autocorrelation structure was used to account for within subject correlation of having repeated measures on the same subject over time. A piecewise linear regression allowing for different slopes between pre-study and study periods was used to estimate the changes on the trajectories due to our intervention. Generalized estimating equation was used to model the differences in treatment burden scaled scores between enrollment and end of study.

\section{3 | RESULTS}

Thirty-nine subjects were enrolled in the study. The mean age of the participants was $15.89 \pm 2.18$ years and $54 \%$ were female. The baseline demographic characteristics are shown in Table 1. Average training time was $29.10 \mathrm{~min}$, including time spent practicing with the device. Participants spent an average of 48.28 weeks \pm 9.28 weeks (range 9-60 weeks) in the study. Mean adherence to weekly spirometry monitoring was $59.47 \pm 24.60 \%$ (range $19.44-100 \%$ ). Nine subjects did not complete the study but were included in the final analysis. These nine subjects spent at average of 36.67 weeks \pm 13.43 weeks (range 9-52 weeks) in the study. Mean adherence to the weekly spirometry monitoring in this group was $36.58 \% \pm 21.47 \%$ (range $17.78-77.78 \%$ ). Of the 30 subjects that completed the study, nine (30\%) had greater than $80 \%$ adherence to the weekly spirometry monitoring.

There was good reliability between the FEV1\% predicted values generated on the Spiro $P D ®$ and those obtained in clinic. A comparison of the mean FEV1\% predicted values obtained at the enrollment and quarterly clinic visits with those generated on the Spiro $P D \circledR$ at or around the same time is shown in Figure 1. The intra-class correlation (ICC) between device and clinic values across all visits was high at 0.80 . More specifically, the ICC was 0.90 at enrollment, 0.82 at 3 months, 0.69 at 6 months, 0.99 at 9 months, and 0.95 at 12 months.

There was a significant improvement in adherence across all four medications during the study as compared to the year prior $(P=0.04)$. Table 2 lists the MPR as a percentage for each of the medications in the 
TABLE 1 Baseline demographics of the study participants

\begin{tabular}{ll} 
Characteristics & $\begin{array}{l}\text { Intervention group } \\
(\mathrm{N}=39) \text { mean (SD) }\end{array}$ \\
\hline Age (years) & $15.89(2.18)$ \\
\hline$\%$ female & 54.00 \\
\hline $\begin{array}{l}\text { Forced expiratory volume in } \\
1 \mathrm{~s} \text { (\% predicted) }\end{array}$ & $87.51(19.80)$ \\
\hline $\begin{array}{l}\text { Body mass index (percentile) } \\
\text { No. of pulmonary exacerbations } \\
\text { (12 months prior) }\end{array}$ & $55.23(25.02)$ \\
\hline
\end{tabular}

year prior to enrollment and during the study. MPR for all medications increased during the study period but the change in MPR was similar across all medications $(P=0.99)$.

Mean treatment burden scaled score on the CFQ-R was 68 at enrollment and 66 at the end of the study $(P=0.14)$. Mean number of pulmonary exacerbations requiring oral or intravenous antibiotics was 1.90 during the study and in the year prior. Mean FEV1\% predicted declined from $88 \%$ to $86 \%$ during the study $(P<0.001)$. However, there were no significant differences in the rate of decline in FEV1\% predicted during the study as compared to that in the year prior $(P>0.05)$. There was also a significant decline in the BMI percentile from $55 \%$ to $52 \%$ during the study $(P=0.009)$. Moreover, the rate of decline in $\mathrm{BMI}$ was higher during the study as compared to that in the year prior $(P=0.031)$. However, there was no correlation between medication adherence during the study and change in treatment burden scaled scores, FEV1\% predicted or BMI percentile $(P>0.05)$.

\section{4 | DISCUSSION}

This was a prospective interventional study evaluating the use of frequent home spirometry to improve medication adherence among adolescents with CF. Subjects monitored their PFTs weekly from home for one-year using the Spiro PD®. Adherence to two oral and two nebulized medications were assessed using prescription refill data. Medication adherence during the study was compared to that in the previous year.

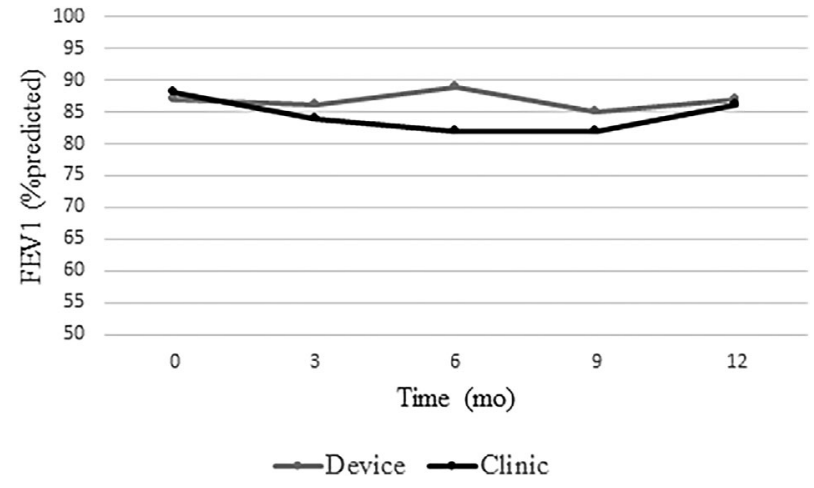

FIGURE 1 Comparison of mean FEV1\% predicted from clinic with that generated on the Spiro $\mathrm{PD} \otimes$
TABLE 2 Medication possession ratio (MPR) by medication type in the year prior to enrollment and during the study

\begin{tabular}{llll} 
Medication name & Pre-study MPR & Study MPR & P-value \\
\hline CF vitamins & $56 \%$ & $61 \%$ & 0.245 \\
Dornase alfa & $66 \%$ & $71 \%$ & 0.188 \\
\hline Pancreatic enzymes & $55 \%$ & $59 \%$ & 0.247 \\
\hline Inhaled hypertonic saline & $66 \%$ & $72 \%$ & 0.125 \\
\hline Across all medications & $60 \%$ & $65 \%$ & 0.038 \\
\hline
\end{tabular}

The participants in this study were younger than those in the Lechtzin et al ${ }^{37}$ home spirometry study (15.89 vs 27.1 years) but could, nevertheless, be readily trained to measure their PFTs from home. They were also able to maintain the weekly monitoring for a full year, similar to the adults in the Lechtzin et $\mathrm{al}^{37}$ study. A pilot study of the Spiro PD ${ }^{\circledR}$ device demonstrated that it could be successfully used by patients with $\mathrm{CF}$ as young as 11 years of age. ${ }^{17}$ Although the training time of 29 min might not be feasible in a busy clinic setting, the participants were trained in the PFT lab after their clinic visit. Moreover, the $29 \mathrm{~min}$ also included time spent practicing with the device with the RT still present to answer questions, which was also made possible by moving the training away from the clinic area. The Spiro $\mathrm{PD} \circledast$ does come with an instructional video, which could also be used in the event of a time or space constraint.

The participants in this study were more adherent to the home spirometry monitoring than the adults in the Lechtzin et $\mathrm{al}^{37}$ study. While in that study $50 \%$ of the participants used the device once a week and only $19 \%$ used the device as instructed for greater than $80 \%$ of the study duration, the participants in this study had a mean adherence rate of $59.47 \%$ and $30 \%$ used the device as instructed for more than $80 \%$ of the study. ${ }^{37}$ One explanation for the better adherence seen in the participants in this study might be the fact that they were asked to measure their PFTs only once a week. Parental supervision may have also contributed to the higher adherence rates seen in our population. However, Modi et $\mathrm{al}^{22}$ reported a gradual decline in parental supervision during the teenage years such that for those who were 15 years of age, only about $10 \%$ of their medical care was supervised by either parent. Perceiving the home spirometry monitoring as adding to the treatment burden might also influence adherence. While the participants in this study did not experience a significant worsening in their perceptions of treatment burden, those who were randomized to the home spirometry arm in the Lechtzin et $\mathrm{al}^{37}$ study did. This difference might be due to the varying demands placed on the participants of the respective studies. Perceptions of treatment burden may also be higher in adults with CF compared to adolescents due to corresponding differences in treatment complexity. ${ }^{27}$

Participants in this study had better medication adherence, even at baseline, than what has been previously reported in this patient population. ${ }^{2,3,4-7}$ Despite that, there was a statistically significant improvement in adherence across all four medications during the study as compared to the year prior. It is possible that this improvement in adherence was due to the fact that this was a study monitoring 
adherence. In a study by Zindani et $\mathrm{al}^{8}$, knowing that their adherence was being monitored (through electronic pill caps and empty nebulizer vials), resulted in adherence rates of $57 \%$ to vitamins and $78 \%$ to dornase alfa in the 12 years and older age group. In a different study of school-aged children, adherence to pancreatic enzymes monitored via electronic pill caps was $38 \%{ }^{7}$ While it is not known how adherent the participants in these different studies were prior to their participation in those studies, the subjects in this study had an adherence rate of $56 \%$ to vitamins, $55 \%$ to pancreatic enzymes, $66 \%$ to dornase alfa, and IHS prior to enrollment. Although the telephone calls were carefully scripted to not discuss medication adherence, it is possible that they also contributed to the improvements in adherence.

Despite the improvements in medication adherence, there was no change in frequency of pulmonary exacerbations or in the rate of decline in FEV1\% predicted. These findings were similar to that in the Lechtzin et $\mathrm{al}^{37}$ study in that even they did not find a significant difference in the rate of decline in FEV1\% predicted between the two groups. The reduction in FEV1\% predicted observed in this study was lower than that seen by Lechtzin et $\mathrm{al}^{37}$ $(-3.58 \%$ vs $-2 \%)$, but this might be due to the younger age of the participants in this study. More importantly, even with the decline, the participants continued to maintain normal PFT. Unlike the Lechtzin et al study, ${ }^{37}$ the participants in this study experienced a decline in BMI percentile. Furthermore, it was at a rate that was higher than that in the previous year. One explanation could be the small sample size. Even with the decline, the mean BMI remained above the 50th percentile.

This study had several limitations starting with its small sample size. Having the participants serve as their own historic controls is also a limitation since there could be changes in adherence with age and worsening disease severity that are independent of the proposed intervention. Another limitation was that adherence was calculated using prescription refill data and this does not necessarily reflect whether an individual is taking the medication as instructed. Furthermore, adherence could be falsely low, particularly for pancreatic enzymes, due to factors such as overfilling. However, prescription refill histories provide an objective assessment of adherence, are less expensive and are more readily available than electronic medication monitoring devices. Another limitation was that the selection process did not include factors such as PFTs, nutritional status or baseline adherence. All eligible individuals who were willing to participate were recruited for the study. The study participants had normal PFTs and BMI and a good adherence rate even in the year prior to enrollment, which might have contributed to the lack of a more robust response to the intervention. The selection process also did not take the transition readiness program that many of the subjects were participating in into account. Since that also addresses issues like medication adherence and selfmanagement, it could have influenced the results of this study. Last, the nine subjects that did not complete the study did not fill out a final CFQ-R, making it difficult to comment on whether treatment burden played a role in their decision to not follow through with the study.
To our knowledge, this was the first study to use frequent home spirometry to successfully improve medication adherence among adolescents with CF. Home spirometry can be readily implemented even in a busy clinic practice. This study used the weekly telephone calls to check on potential difficulties with the device but they could certainly be used to provide real-time feedback on the spirometry results and that might potentially improve medication adherence even more. Text messages are another less time-consuming alternative and one that was preferred by several of the study participants. More studies with larger sample sizes are needed to further explore the benefits of frequent home spirometry in improving adherence to treatment, quality of life, and health outcomes in this vulnerable patient population.

\section{ACKNOWLEDGMENTS}

The study was supported by the Cystic Fibrosis Foundation Third Year Fellowship Training Grant (SHAKKO15A0). Presented in part at the American Thoracic Society Meeting in Washington DC, May 23rd, 2017 and at the North American Cystic Fibrosis Conference in Indianapolis, IN, November 2nd, 2017.

\section{ORCID}

Samya Z. Nasr (iD http://orcid.org/0000-0002-4124-316X

\section{REFERENCES}

1. Narayanan S, Mainz JG, Gala S, Tabori H, Grossoehme D. Adherence to therapies in cystic fibrosis: a targeted literature review. Expert Rev Respir Med. 2017;11:129-145.

2. Shakkottai A, Kidwell KM, Townsend M, Nasr SZ. A five-year retrospective analysis of adherence in cystic fibrosis. Pediatr Pulmonol. 2015;50:1224-1229.

3. Quittner AL, Zhang J, Marynchenko M, et al. Pulmonary medication adherence and health-care use in cystic fibrosis. Chest. 2014;146: 142-151.

4. Eakin MN, Bilderback A, Boyle MP, Mogayzel PJ, Riekert KA. Longitudinal association between medication adherence and lung health in people with cystic fibrosis. J Cyst Fibros. 2011;10: 258-264.

5. Nasr SZ, Chou W, Villa KF, Chang E, Broder MS. Adherence to dornase alfa treatment among commercially insured patients with cystic fibrosis. J Med Econ. 2013;16:801-808.

6. Modi AC, Lim CS, Yu N, Geller D, Wagner MH, Quittner AL. A multi-method assessment of treatment adherence for children with cystic fibrosis. J Cyst Fibros. 2006;5:177-185.

7. Barker DH, Quittner AL. Parental depression and pancreatic enzymes adherence in children with cystic fibrosis. Pediatrics. 2016;137: e20152296.

8. Zindani GN, Streetman DD, Streetman DS, Nasr SZ. Adherence to treatment in children and adolescent patients with cystic fibrosis. J Adolesc Health. 2006;38:13-17.

9. Siracusa CM, Ryan J, Burns L, et al. Electronic monitoring reveals highly variable adherence patterns in patients prescribed ivacaftor. J Cyst Fibros. 2015;14:621-626.

10. Ball R, Southern KW, McCormack P, Duff AJ, Brownlee KG, McNamara PS. Adherence to nebulised therapies in adolescents with cystic fibrosis is best on week-days during school term-time. J Cyst Fibros. 2013;12:440-444. 
11. Bishay LC, Sawicki GS. Strategies to optimize treatment adherence in adolescent patients with cystic fibrosis. Adolesc Health Med Ther. 2016;7:117-124.

12. Rosenfeld $M$, VanDevanter $D R$, Ren $C L$, et al. Investigators of coordinators of the epidemiologic study of cystic fibrosis. Decline in lung function does not predict future decline in lung function in cystic fibrosis patients. Pediatr Pulmonol. 2015;50:856-862.

13. Cogen J, Emerson J, Sanders DB, et al. EPIC study group. Risk factors for lung function decline in a large cohort of young cystic fibrosis patients. Pediatr Pulmonol. 2015;50:763-770.

14. McPhail GL, Acton JD, Fenchel MC, Amin RS, Seid M. Improvements in lung function outcomes in children with cystic fibrosis are associated with better nutrition, fewer chronic pseudomonas aeruginosa infections, and dornase alfa use. J Pediatr. 2008;153:752-757.

15. Bucks RS, Hawkins K, Skinner TC, Horn S, Seddon P, Horne R. Adherence to treatment in adolescents with cystic fibrosis: the role of illness perceptions and treatment beliefs. J Pediatr Psychol. 2009;34: 893-902.

16. Sawicki GS, Heller KS, Demars N, Robinson WM. Motivating adherence among adolescents with cystic fibrosis: youth and parent perspectives. Pediatr Pulmonol. 2015;50:127-136.

17. Shakkottai A, Nasr SZ. The use of home spirometry in pediatric cystic fibrosis patients: results of a feasibility study. Glob Pediatr Health. 2017;4:1-6.

18. Dziuban EJ, Saab-Abazeed L, Chaudhry SR, Streetman DS, Nasr SZ. Identifying barriers to treatment adherence and related attitudinal patterns in adolescents with cystic fibrosis. Pediatr Pulmonol. 2010;45:450-458.

19. George M, Rand-Giovannetti D, Eakin MN, Borrelli B, Zettler M, Riekert KA. Perceptions of barriers and facilitators: self-management decisions by older adolescents and adults with CF. J Cyst Fibros. 2010;9:425-432.

20. Goodfellow NA, Hawwa AF, Reid AJ, Horne R, Shields MD, McElnay JC. Adherence to treatment in children and adolescents with cystic fibrosis: a cross-sectional, multi-method study investigating the influence of beliefs about treatment and parental depressive symptoms. BMC Pulm Med. 2015;15:43.

21. Butcher JL, Nasr SZ. Direct observation of respiratory treatments in cystic fibrosis: parent-child interactions relate to medical regimen adherence. J Pediatr Psychol. 2015;40:8-17.

22. Modi AC, Marciel KK, Slater SK, Drotar D, Quittner AL. The influence of parental supervision on medical adherence in adolescents with cystic fibrosis: developmental shifts from pre to late adolescence. Children's Health Care. 2008;37:78-92.

23. Faint NR, Staton JM, Stick SM, Foster JM, Schultz A. Investigating selfefficacy, disease knowledge and adherence to treatment in adolescents with cystic fibrosis. J Paediatr Child Health. 2017;53:488-493.

24. Bregnballe V, Schiøtz PO, Boisen KA, Pressler T, Thastum M. Barriers to adherence in adolescents and young adults with cystic fibrosis: a questionnaire study in young patients and their parents. Patient Prefer Adherence. 2011;5:507-515.

25. Quittner AL, Saez-Flores E, Barton JD. The psychological burden of cystic fibrosis. Curr Opin Pulm Med. 2016;22:187-191.

26. Sawicki GS, Goss $\mathrm{CH}$. Tackling the increasing complexity of $\mathrm{CF}$ care. Pediatr Pulmonol. 2015;50:S74-S79.
27. Sawicki GS, Ren CL, Konstan MW, Millar SJ, Pasta DJ, Quittner AL. Investigators and Coordinators of the Epidemiologic Study of Cystic Fibrosis. Treatment complexity in cystic fibrosis: trends over time and associations with site-specific outcomes. J Cyst Fibros. 2013;12: 461-467.

28. Modi AC, Pai AL, Hommel KA, et al. Pediatric self-management: a framework for research, practice, and policy. Pediatrics. 2012;129: e473-e485.

29. Drotar D. Strategies of adherence promotion in the management of pediatric chronic conditions. J Dev Behav Pediatr. 2013;34:716-729.

30. Geller DE, Madge S. Technological and behavioral strategies to reduce treatment burden and improve adherence to inhaled antibiotics in cystic fibrosis. Respir Med. 2011;105:S24-S31.

31. Johnson KB, Culpepper D, Scott P, Gordon JS, Harris C. The utility of providing automated medication dose reminders to young children on chronic medication. J Telemed Telecare. 2011;17:387-391.

32. Morton RW, Elphick HE, Edwards E, Daw WJ, West NS. Investigating the feasibility of text message reminders to improve adherence to nebulized medication in children and adolescents with cystic fibrosis. Patient Prefer Adherence. 2017;11:861-869.

33. Marciel KK, Saiman L, Quittell LM, Dawkins K, Quittner AL. Cell phone intervention to improve adherence: cystic fibrosis care team, patient, and parent perspectives. Pediatr Pulmonol. 2010;45:157-164.

34. Narayan O, Davies S, Tibbins C, Rees JM, Lenney W, Gilchrist FJ. Developing a handheld record for patients with cystic fibrosis. Patient Relat Outcome Meas. 2015;6:225-228.

35. Hilliard ME, Hahn A, Ridge AK, Eakin MN, Riekert KA. User preferences and design recommendations for an mHealth app to promote cystic fibrosis self-management. JMIR Mhealth Uhealth. 2014;2:e44.

36. Finkelstein SM, Wielinski CL, Kujawa SJ, Loewenson R, Warwick WJ. The impact of home monitoring and daily diary recording on patient status in cystic fibrosis. Pediatr Pulmonol. 1992;12:3-10.

37. Lechtzin N, Mayer-Hamblett N, West NE, et al. EICE study team. home monitoring in $\mathrm{CF}$ to identify and treat acute pulmonary exacerbations: EICE study results. Am J Respir Crit Care Med. 2017;196:1144-1151.

38. Borowitz D, Baker RD, Stallings V. Consensus report on nutrition for pediatric patients with cystic fibrosis. J Pediatr Gastroenterol Nutr. 2002;35:246-259.

39. Mogayzel PJ, Jr, Naureckas ET, Robinson KA, et al. Pulmonary Clinical Practice Guidelines Committee. Cystic fibrosis pulmonary guidelines. Chronic medications for maintenance of lung health. Am J Respir Crit Care Med. 2013;187:680-689.

40. Quittner AL, Sawicki GS, McMullen A, et al. Psychometric evaluation of the cystic fibrosis questionnaire-revised in a national sample. Qual Life Res. 2012;21:1267-1278.

How to cite this article: Shakkottai A, Kaciroti N, Kasmikha L, Nasr SZ. Impact of home spirometry on medication adherence among adolescents with cystic fibrosis. Pediatric Pulmonology. 2018;53:431-436. https://doi.org/10.1002/ppul.23950 